\title{
Proportional Relations Between Systolic, Diastolic and Mean Pulmonary Artery Pressure are Explained by Vascular Properties
}

\author{
Taco Kind, ${ }^{1}$ Theo J. C. Faes, ${ }^{2}$ Anton Vonk-Noordegraaf, ${ }^{1}$ and Nico Westerhof ${ }^{1,3}$ \\ ${ }^{1}$ Department of Pulmonary Diseases, Institute for Cardiovascular Research, VU University Medical Center, Amsterdam, \\ The Netherlands; ${ }^{2}$ Department of Physics and Medical Technology, Institute for Cardiovascular Research, VU University \\ Medical Center, Amsterdam, The Netherlands; and ${ }^{3}$ Department of Physiology, Institute for Cardiovascular Research, \\ VU University Medical Center, Amsterdam, The Netherlands
}

(Received 6 August 2010; accepted 22 October 2010; published online 11 November 2010)

Associate Editor Steven C. George oversaw the review of this article.

\begin{abstract}
Recently, it was shown that proportional relationships exist between systolic, diastolic and mean pulmonary artery pressure $\left(P_{\text {sys }}, P_{\text {dia }}\right.$ and $\left.P_{\text {mean }}\right)$ and that they are maintained under various conditions in both health and disease. An arterial-ventricular interaction model was used to study the contribution of model parameters to the ratios $P_{\text {sys }} / P_{\text {mean }}$, and $P_{\text {dia }} / P_{\text {mean }}$. The heart was modeled by a timevarying elastance function, and the arterial system by a threeelement windkessel model consisting of peripheral resistance, $R_{\mathrm{p}}$, arterial compliance $C_{\mathrm{a}}$, and pulmonary artery characteristic impedance $Z_{0}$. Baseline model parameters were estimated in control subjects and compared to values estimated in patients with pulmonary hypertension. Results indicate that experimentally derived ratios $P_{\text {sys }} / P_{\text {mean }}$ and $P_{\text {dia }} / P_{\text {mean }}$ could be accurately reproduced using our model (1.59 and 0.61 vs. 1.55 and 0.64 , respectively). Sensitivity analysis showed that the (empirical) constancy of $P_{\text {sys }} / P_{\text {mean }}$ and $P_{\text {dial }} /$ $P_{\text {mean }}$ was primarily based on the inverse hyperbolic relation between total vascular resistance $\left(R_{\mathrm{T}}\right.$; calculated as $\left.R_{\mathrm{p}}+Z_{0}\right)$ and $C_{\mathrm{a}}$, (i.e. constant $R_{\mathrm{T}} C_{\mathrm{a}}$ product). Of the cardiac parameters, only heart rate affected the pressure ratios, but the contribution was small. Therefore, we conclude that proportional relations between systolic, diastolic and mean pulmonary artery pressure result from the constancy of $R_{\mathrm{T}} C_{\mathrm{a}}$ thus from pulmonary arterial properties, with only little influence of heart rate.
\end{abstract}

Keywords-Pulmonary artery, Pressure, Proportional pressure, Elastance model, Three-element windkessel model.

\section{INTRODUCTION}

It has been shown that proportional relationships exist between systolic, diastolic and mean pulmonary

Address correspondence to Taco Kind, Department of Pulmonary Diseases, Institute for Cardiovascular Research, VU University Medical Center, Amsterdam, The Netherlands. Electronic mail: t.kind@vumc.nl artery pressure, and that they are preserved under various conditions in both health and disease., $3,6,23$ Although these empirical relations have shown to be of practical relevance, the physiological mechanism underlying these relations has not yet been elucidated. Since pressure is the result of the interaction between right heart and lung circulation, these proportional relations may, in principle, be explained by ventricular and vascular properties contributing to pulmonary artery pressure.

Recently, Lankhaar et al. ${ }^{8,9}$ showed in a cohort of normal subjects and patients with pulmonary arterial hypertension (PAH) before and after treatment, that pulmonary resistance $(R)$ and compliance $(C)$ are related by an inverse hyperbolic relationship. This inverse relation might, in part, be the underlying mechanism of the proportional relations between the systolic, diastolic and mean pressure.

The aim of this study is to investigate how the proportionality of the pulmonary artery pressure components depends on cardiac and arterial parameters, and, in particular, the inverse relation between $R$ and $C$, using an interaction model of the pulmonary circulation. ${ }^{17,20}$

\section{METHODS}

\section{Mathematical Model of the Pulmonary Circulation}

Pulmonary artery pressure $P_{\mathrm{a}}(t)$ was computed using an arterial-ventricular interaction model (Fig. 1a). ${ }^{17,20}$ The arterial bed was modeled using a three-element lumped-parameter windkessel model ${ }^{8,25,26}$ consisting of characteristic impedance of the proximal pulmonary artery $\left(Z_{0}\right)$ and a combination of peripheral pulmonary 
(a)
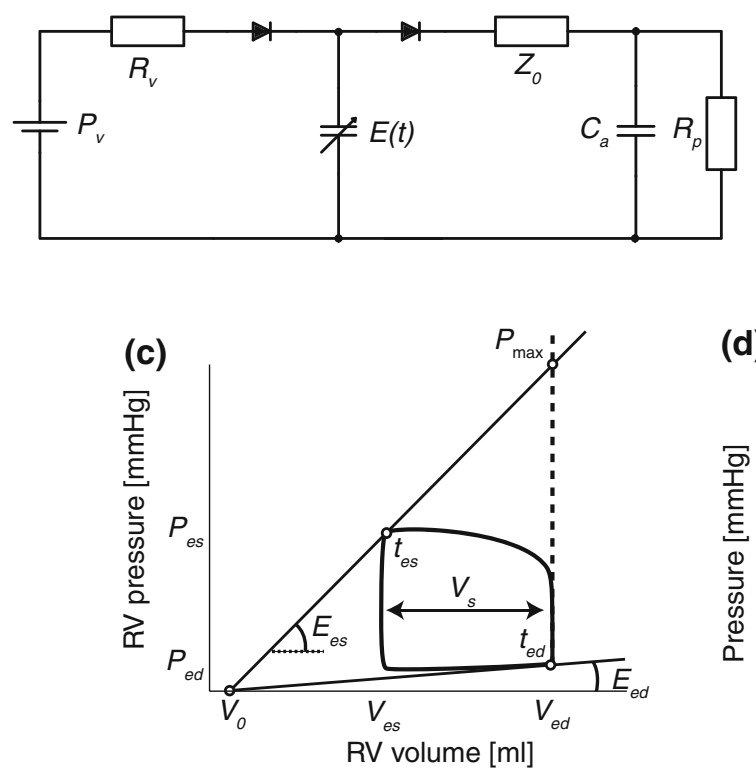

(b)

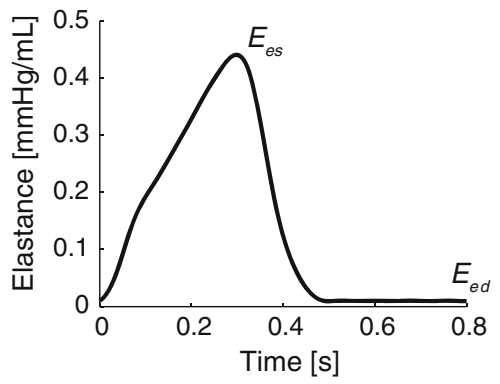

(d)

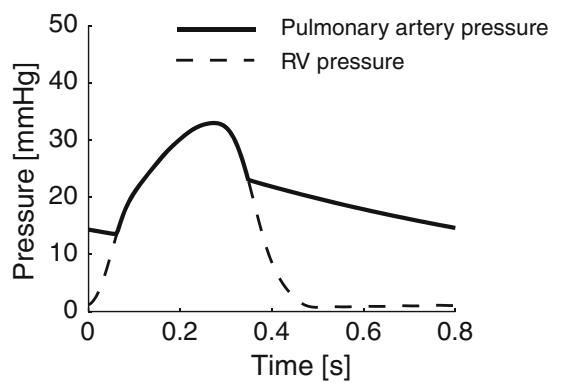

FIGURE 1. (a) Arterial-ventricular interaction model. The arterial system is modeled by a three-element windkessel model, consisting of characteristic impedance $\left(Z_{0}\right)$, peripheral vascular resistance $\left(R_{\mathrm{p}}\right)$ and arterial compliance $\left(C_{\mathrm{a}}\right)$. The heart is modeled using a time-varying elastance model, with elastance function constructed using a normalized elastance curve ${ }^{18}$ as depicted in (b). A pressure-volume loop with the major ventricular parameters is shown in (c). Examples of simulated pulmonary artery pressure $P_{\mathrm{a}}(t)$ and right ventricular pressure $P_{\mathrm{RV}}(t)$ are shown in (d). $E_{\mathrm{es}}=$ end-systolic elastance; $E_{\mathrm{ed}}=$ end-diastolic elastance; $V_{0}=$ intercept volume; $P_{\mathrm{v}}=$ venous filling pressure; $R_{\mathrm{v}}=$ venous resistance; $t_{\mathrm{es}}=$ time to reach end-systolic elastance; $t_{\mathrm{ed}}=$ time to reach end-diastolic elastance; $V_{\mathrm{s}}=$ stroke volume; $\boldsymbol{P}_{\max }=$ maximum isovolumic pressure.

resistance $\left(R_{\mathrm{p}}\right)$ and pulmonary arterial compliance $\left(C_{\mathrm{a}}\right)$ (Fig. 1a). Right ventricular (RV) function was modeled using a linear time-varying elastance model, ${ }^{21}$ including a description of the normalized elastance as a function of time as proposed by Senzaki et al. ${ }^{18}$ (Fig. 1a). Although originally introduced for the left ventricle, the elastance model is assumed to be valid for the RV. ${ }^{10}$

Elastance is parameterized by end-systolic elastance $\left(E_{\mathrm{es}}\right)$, end-diastolic elastance $\left(E_{\mathrm{ed}}\right)$, intercept volume $\left(V_{0}\right)$ of the pressure-volume relationship, heart rate $(H R)$, time to reach end-systolic elastance $\left(t_{\mathrm{es}}\right)$. Diastolic filling of the ventricle is described with a venous filling pressure of the $\mathrm{RV}\left(P_{\mathrm{v}}\right)$, and a small venous resistance $\left(R_{\mathrm{v}}\right)$ over which filling takes place. The tricuspid and pulmonary valves were assumed sufficient. Left atrial pressure $\left(P_{\text {la }}\right)$ was neglected in the model (set at zero). The model directly yields pulmonary arterial and ventricular pressure (Fig. 1d).

\section{Model Parameters and Simulations}

Model parameters were estimated from data measured in a group of 11 control subjects (Group 1; see paragraph patient selection) and then averaged to obtain a single set of baseline parameters. Arterial parameters, $Z_{0}, R_{\mathrm{p}}$ and $C_{\mathrm{a}}$, were estimated from measured pressure and flow using a prediction-error minimization method. Instead of averaging $C_{\mathrm{a}}$, baseline $C_{\mathrm{a}}$ was determined by averaging $1 / C_{\mathrm{a}}$ followed by calculating the reciprocal. This procedure was performed because of the inverse relation between resistance and compliance. Elastance parameters were obtained as follows (see also Fig. 1c). End-systolic elastance was calculated as $E_{\mathrm{es}}=\left(P_{\max }-P_{\mathrm{es}}\right) / V_{\mathrm{s}}$, with $P_{\max }$ maximum isovolumic pressure, $P_{\mathrm{es}}$ endsystolic pulmonary artery pressure, and $V_{\mathrm{s}}$ stroke volume. $P_{\max }$ was computed using a single-beat estimation method ${ }^{2,22,24} ; P_{\mathrm{es}}$ was determined at endsystole, $t_{\mathrm{es}}$, obtained from pulmonary artery flow $F_{\mathrm{a}}(t)$ as the time that flow reaches zero. Note that the corresponding pressure is somewhat lower than the value obtained from a pressure-volume loop (upper left corner). Therefore, baseline $E_{\mathrm{es}}$ is slightly lower compared to $E_{\text {es }}$ obtained from a pressure-volume loop.

The $F_{\mathrm{a}}(t)$ tracings were also used to derive stroke volume, $V_{\mathrm{s}}$. Subsequently, an approximation of $V_{0}$ was made using the $E_{\mathrm{es}}=P_{\mathrm{es}} /\left(V_{\mathrm{es}}-V_{0}\right)$, with $V_{\mathrm{es}}$ derived from MR imaging (see paragraph patient selection and measurements). Diastolic pressure of the RV $\left(P_{\mathrm{v}}\right)$ was assumed to be equal to mean right atrial pressure and $E_{\text {ed }}$ was then estimated by $P_{\mathrm{v}} /\left(V_{\text {ed }}-V_{0}\right)$, with $V_{\text {es }}$ also derived from MR imaging. The 'venous' resistance $\left(R_{\mathrm{v}}\right)$ was arbitrarily set at $0.1 \mathrm{mmHg} \mathrm{s} / \mathrm{mL}^{20}$ 
Numerical integration was performed using the MATLAB ordinary differential equations solver ODE15S (R14SP1), supporting stiff differential equations and a variable order method. For each simulation run, 25 heartbeats were simulated to obtain a steady state, and only the last beat was taken to obtain a single pulmonary artery pressure curve (Fig. 1d).

\section{Data Analysis}

\section{Proportional Pressure Relations}

Previously, proportional relations have been reported between systolic $\left(P_{\text {sys }}\right)$ and mean $\left(P_{\text {mean }}\right)$ pressure, ${ }^{3}$ and between diastolic $\left(P_{\text {dia }}\right)$ and systolic $\left(P_{\text {sys }}\right)$ with mean pressure. ${ }^{23}$ The reported intercepts of these linear relations were very small (i.e. ranging -0.22 to $2 \mathrm{mmHg}$ for $P_{\text {sys }}$ and $P_{\text {mean }}{ }^{3,6,23}$ and -0.66 for $P_{\text {dia }}$ and $P_{\text {mean }}{ }^{23}$ ) in comparison to the range of systolic and diastolic pressures. Thus, these numerically insignificant intercepts can be neglected in a first order approximation, and pressure components can therefore be considered not only linearly related, but also proportionally related.

In this study, the proportionalities are expressed by the ratios $K_{\text {sys }}=P_{\text {sys }} / P_{\text {mean }}$ and $K_{\text {dia }}=P_{\text {dia }} / P_{\text {mean }}$ and were tested in both our measured and simulated data and, moreover, were compared to data reported in literature.

\section{Definition of the $R C$-time}

To assess the hypothesized association between the inverse proportional RC-relation (i.e. constant RC-time $)^{8,9,11,14}$ and the pressure proportionalities, the definition of RC-time requires some attention.

The three-element windkessel model consists of two resistive components, peripheral resistance $\left(R_{\mathrm{p}}\right)$ and characteristic impedance, which is also modeled as a resistance $\left(Z_{0}\right)$, thus total pulmonary vascular resistance $\left(R_{\mathrm{T}}\right)$ equals the summation of these components. The constant product not only holds for $R_{\mathrm{p}}$ and $C_{\mathrm{a}}{ }^{8}$ but also for $R_{\mathrm{T}}$ and $C_{\mathrm{a}} \cdot{ }^{9,13,15}$ The product $R_{\mathrm{p}}$ times $C_{\mathrm{a}}$ determines diastolic pressure decay, whereas the product $R_{\mathrm{T}}$ times $C_{\mathrm{a}}$ also determines systolic pressure loading. Moreover, $Z_{0}$ primarily affects $P_{\text {sys }}$, while $R_{\mathrm{p}}$ both affects $P_{\text {mean }}, P_{\text {sys }}$ and $P_{\text {dia }}$. In this study we considered the product of total resistance and compliance (i.e. $R_{\mathrm{T}}$ times $C_{\mathrm{a}}$ ).

A drawback of the three-element windkessel model is that the characteristic impedance is not an Ohmic resistance but an impedance not existing for mean pressure and flow. In this model the magnitude (modulus) is constant with value $\left|Z_{0}\right|$ for all frequencies. Thus, for low frequencies total impedance of the arterial system is described less accurate. In the physiological range, however, the errors in systolic and diastolic pressure are small as has been shown by Stergiopulos et al. ${ }^{20}$ Moreover, being a lumped model, it does not contain spatial information and therefore cannot describe wave reflection phenomena. ${ }^{20}$

\section{Contribution of Arterial and Cardiac Changes to $K_{\text {sys }}$ and $K_{\text {dia }}$}

Sensitivity analysis was performed to study the effect of isolated alterations in arterial parameters $\left(R_{\mathrm{T}}\right.$ and $\left.C_{\mathrm{a}}\right)$ and ventricular parameters (HR, $E_{\mathrm{es}}, E_{\mathrm{ed}}, V_{0}$, $\left.t_{\mathrm{es}}, P_{\mathrm{v}}\right)$ on $P_{\mathrm{a}}(t)$, (including $K_{\mathrm{sys}}$ and $\left.K_{\mathrm{dia}}\right)$. In these analyses, parameter values were varied from 50 to $250 \%$ of baseline values as determined from mean values of the control group (Group 1; see paragraph patient selection).

For the arterial parameters, two scenarios were simulated, namely with and without the constraint of a constant RC-time. The rationale of this approach is that experimental data shows constant $K_{\text {sys }}$ and $K_{\text {dia }}$ with obvious large mutual differences in $R_{\mathrm{T}}$ and $C_{\mathrm{a}}$ but constant $R_{\mathrm{T}} C_{\mathrm{a}}$, and sensitivity analysis using the model should reproduce this constancy. In the first scenario, it was assumed that no proportional relation exists between $R_{\mathrm{T}}$ and $C_{\mathrm{a}}$. Thus, the individual effect of varying $R_{\mathrm{T}}$ or $C_{\mathrm{a}}$ on $K_{\mathrm{sys}}$ and $K_{\mathrm{dia}}$ were determined. For the second data set, it was assumed that $R_{\mathrm{T}} C_{\mathrm{a}}$ is constant. Hence, an increase in $R_{\mathrm{T}}$ is accompanied by a proportional decrease in $C_{\mathrm{a}}$ (or vice versa) and the combined effect on $K_{\text {sys }}$ and $K_{\text {dia }}$ was studied.

\section{Patient Selection and Measurements}

The institutional ethics committee approved the study and all patients gave informed consent. Included were 11 patients suspected of pulmonary hypertension at echocardiography but in whom the diagnosis could not be established. These patients served as control subjects and were used to compute baseline model parameters (Group 1). We also included 98 patients (Group 2) diagnosed with PAH. In 19 of these patients the complete set of model parameters was computed for comparison purposes (Group 2a). In the remainder of patients (Group 2b), we only determined $P_{\text {sys }}, P_{\text {dia }}$, $P_{\text {mean }}$, the ratios $K_{\text {sys }}$ and $K_{\text {dia }}$ and heart rate.

All patients were evaluated according to a standard diagnostic protocol. Pressure was measured with a 6-Fr fluid-filled, single-lumen, multipurpose catheter (Cordis, Miami Lakes, FL) in the main pulmonary artery and in the right ventricle. All measurements were carried out after careful flushing and use of heparin to prevent catheter artifacts.

Pressure tracings of non-PH patients and subset of $\mathrm{PAH}$ patients were digitally stored using an $\mathrm{AD}$ 
instruments PowerLab acquisition system. These patients also underwent cardiac MR imaging for flow and volume measurements within 1 day before or after right heart catheterization. Despite this time difference in the measurements, it was assumed that the patient was in the same hemodynamic steady state. MR examination was performed on a 1.5-T Siemens Avanto MRI system (Siemens Medical Solutions, Germany), equipped with a 6-element phased-array coil. Main pulmonary artery flow was measured using phase-contrast velocity quantification. A twodimensional spoiled gradient-echo pulse sequence was applied with an excitation angle of $15^{\circ}$, a TE of $4.8 \mathrm{~ms}$, a TR of $11 \mathrm{~ms}$, and a receiver bandwidth of $170 \mathrm{~Hz}$ per pixel. Velocity sensitivity was initially set to $150 \mathrm{~cm} / \mathrm{s}$, but adjusted to lower or higher values in individual cases. Velocity encoding was interleaved resulting in a temporal resolution of $22 \mathrm{~ms}$. Field of view was set to $260 \times 320 \mathrm{~mm}$, and the matrix size was set to $208 \times 256$. Pulmonary flow was obtained using Medis Flow software package (Medis, Leiden, The Netherlands). Contours around the pulmonary artery cross section were semi-automatically drawn in the MRI magnitude images. The average velocity within each contour was multiplied with its area to obtain a volumetric flow curve as a function of time.

End-diastolic volume was obtained from a stack of short-axis slices with a typical slice thickness of $5 \mathrm{~mm}$ and an interslice gap of $5 \mathrm{~mm}$, fully covering both ventricles from base to apex. Endocardial surfaces, excluding trabeculae and papillary muscles, were manually traced using Medis Mass (Medis, Leiden, The Netherlands) to obtain end-diastolic and endsystolic volumes.

\section{Data Pre-processing}

Due to the time-delay between pressure and flow measurements and the different sampling rates $(250 \mathrm{~Hz}$ vs. $45 \mathrm{~Hz}$, respectively) the data were pre-processed. This was performed in a similar manner as in Lankhaar et $a l .{ }^{8}$ Briefly, a stationary pressure interval of about $25 \mathrm{~s}$ with no underdamping artifacts was selected. The selection was filtered using a fifth-order Butterworth (cut-off frequency was $10 \mathrm{~Hz}$ ). Subsequently, end-diastole was identified using the $\mathrm{R}$ wave of the ECG and the cardiac cycles were ensemble averaged. The flow curve was resampled to the sampling rate of pressure.

Synchronization was performed using the following approach. The measured flow and pressure curves were used to estimate a three-element windkessel model and to predict pressure. The error between measured and predicted pressure was determined (i.e., the sum of squared residuals). Flow was then shifted in time and the windkessel model was estimated again to predict pressure. Shifting of the flow curve was repeated until the difference between predicted and measured pressure was minimal.

\section{RESULTS}

\section{Patient Hemodynamics and Model Parameters}

Hemodynamic data and model parameters are summarized in Table 1. Figure 1d shows a simulated pulmonary arterial pressure curve using the arterialventricular interaction model with average parameter values of the control group (Group 1) in Table 1.

\section{Proportional Pressure Components and Inverse RC-relation}

Figure 2a shows the strong relations of diastolic and systolic pressure with mean pressure in the experimental data (control subjects, Group 1, and PAH patients, Groups $2 \mathrm{a}+2 \mathrm{~b}$ ). In the experimental data ratios $K_{\text {sys }}$ and $K_{\text {dia }}$ were found $1.59 \pm 0.15$ and $0.61 \pm 0.09$, respectively. These values were not significantly different from the ratios obtained using the arterial-ventricle model with model parameters set at values of Group 1: $K_{\mathrm{sys}}=1.55$ and $K_{\mathrm{dia}}=0.64$, respectively.

Figure $2 \mathrm{~b}$ shows the strong inverse proportional relation between $R_{\mathrm{T}}$ and $C_{\mathrm{a}}$ for all subjects in Group 1 and $2 \mathrm{a}\left(R^{2}=0.89, p<0.001\right)$, with a RC-time of $0.79 \mathrm{~s}$. Note that the RC-time does not differ significantly in the control subjects (0.82) and PAH patients (0.76; see Table 1$)$.

\section{Sensitivity Analysis}

\section{Arterial Parameters}

Figure 3 shows the effect of isolated changes in arterial parameters on the arterial pressures $\left(P_{\text {sys }}, P_{\text {dia }}\right.$ and $P_{\text {mean }}$; top row) and pressure ratios ( $K_{\text {sys }}$ and $K_{\text {dia }}$; bottom-row), under the condition of a non-constant $R_{\mathrm{T}} C_{\mathrm{a}}$-time (scenario 1) and a constant $R_{\mathrm{T}} C_{\mathrm{a}}$-time (scenario 2). Parameters were varied from 50 to $250 \%$ of baseline values (Group 1; Table 1). The figure depicts that $P_{\text {sys }}, P_{\text {dia }}, P_{\text {mean }}$ vary considerably with varying parameters, as well for $K_{\text {sys }}$ and $K_{\text {dia }}$ in case of a non-constant RC-time. In case of a constant RC-time $K_{\text {sys }}$ and $K_{\text {dia }}$ remain constant supporting the experimental results in Fig. 2a. Note that, with nonconstant $R_{\mathrm{T}} C_{\mathrm{a}}$-time (scenario 1 ) the effects on $K_{\text {sys }}$ and $K_{\text {dia }}$ were reversed but of similar magnitude, while for the constant $R_{\mathrm{T}} C_{\mathrm{a}}$-time (scenario 2 ) the $K_{\text {sys }}$ and $K_{\text {dia }}$ are almost constant. 
TABLE 1. Hemodynamics and model parameters in control subjects and PAH patients.

\begin{tabular}{|c|c|c|c|}
\hline $\begin{array}{l}\text { Group } \\
\text { Parameter }\end{array}$ & $\begin{array}{c}1 \\
\text { Controls }(n=11)\end{array}$ & $\begin{array}{c}2 \mathrm{a} \\
\mathrm{PAH}(n=19)\end{array}$ & $\begin{array}{c}2 \mathrm{~b} \\
\mathrm{PAH}(n=79)\end{array}$ \\
\hline Systolic PA pressure $\left(P_{\text {sys }}\right), \mathrm{mmHg}$ & $32 \pm 6$ & $86 \pm 18^{*}$ & $81 \pm 22^{*}$ \\
\hline Diastolic PA pressure $\left(P_{\mathrm{dia}}\right), \mathrm{mmHg}$ & $13 \pm 4$ & $36 \pm 9^{*}$ & $30 \pm 10^{*}$ \\
\hline Mean PA pressure $\left(P_{\text {mean }}\right), \mathrm{mmHg}$ & $21 \pm 4$ & $55 \pm 11^{*}$ & $50 \pm 13^{*}$ \\
\hline Ratio $K_{\text {sys }}\left(P_{\text {sys }} / P_{\text {mean }}\right)$ & $1.52 \pm 0.15$ & $1.57 \pm 0.13$ & $1.59 \pm 0.16$ \\
\hline Ratio $K_{\text {dia }}\left(P_{\text {dia }} / P_{\text {mean }}\right)$ & $0.58 \pm 0.10$ & $0.65 \pm 0.07$ & $0.59 \pm 0.09$ \\
\hline Characteristic impedance $\left(Z_{0}\right), \mathrm{mmHg} \mathrm{s} / \mathrm{mL}$ & $0.05 \pm 0.01$ & $0.11 \pm 0.05^{*}$ & Not determined \\
\hline Peripheral resistance $\left(R_{\mathrm{p}}\right), \mathrm{mmHg} \mathrm{s} / \mathrm{mL}$ & $0.30 \pm 0.08$ & $0.90 \pm 0.37^{*}$ & Not determined \\
\hline Total vascular resistance $\left(R_{\mathrm{T}}\right), \mathrm{mmHg} \mathrm{s} / \mathrm{mL}$ & $0.32 \pm 0.09$ & $1.01 \pm 0.39^{*}$ & Not determined \\
\hline Arterial compliance $\left(C_{\mathrm{a}}\right), \mathrm{mL} / \mathrm{mmHg}$ & $5.85 \pm 2.71$ & $0.98 \pm 0.53$ * & Not determined \\
\hline Inverse arterial compliance $\left(1 / C_{\mathrm{a}}\right), \mathrm{mmHg} / \mathrm{mL}$ & $0.35 \pm 0.13$ & $1.37 \pm 0.77^{\star}$ & Not determined \\
\hline$R_{\mathrm{T}} C_{\mathrm{a}}$-time, $\mathrm{s}$ & $0.82 \pm 0.24$ & $0.76 \pm 0.29$ & Not determined \\
\hline End-systolic elastance $\left(E_{\mathrm{es}}\right), \mathrm{mL} / \mathrm{mmHg}$ & $0.44 \pm 0.41$ & $1.51 \pm 0.87^{\star}$ & Not determined \\
\hline End-diastolic elastance $\left(E_{\mathrm{ed}}\right), \mathrm{mL} / \mathrm{mmHg}$ & $0.01 \pm 0.03$ & $0.06 \pm 0.06$ & Not determined \\
\hline Intercept volume $\left(V_{0}\right), \mathrm{mL}$ & $-50 \pm 106$ & $71 \pm 87^{\star}$ & Not determined \\
\hline Time to reach $E_{\mathrm{es}}\left(t_{\mathrm{es}}\right), \mathrm{s}$ & $0.33 \pm 0.04$ & $0.35 \pm 0.07$ & Not determined \\
\hline Heart rate $(\mathrm{HR})$, beats/min & $77 \pm 13$ & $83 \pm 15$ & $80 \pm 16$ \\
\hline Filling pressure $\left(P_{\mathrm{v}}\right), \mathrm{mmHg}$ & $6.5 \pm 7.0$ & $7.9 \pm 6.0^{*}$ & Not determined \\
\hline End-diastolic volume $\left(V_{\mathrm{ed}}\right), \mathrm{mL}$ & $103 \pm 64$ & $173 \pm 63^{*}$ & Not determined \\
\hline Peak isovolumic pressure $\left(P_{\max }\right), \mathrm{mmHg}$ & $48 \pm 14$ & $124 \pm 47^{*}$ & Not determined \\
\hline Stroke volume $\left(V_{\mathrm{s}}\right), \mathrm{mL}$ & $68 \pm 17$ & $46 \pm 18^{*}$ & Not determined \\
\hline
\end{tabular}

Values are mean \pm SD. Group 1 consists of non-PH patients and served as the control group (see text). Group 2a consists of $\mathrm{PAH}$ patients in whom all model parameters were estimated for comparison with control values (i.e. to obtain the physiological variation). Group $2 \mathrm{~b}$ consists of $\mathrm{PH}$ patients who were included to increase the total number of patients to compare individual hemodynamical measurements.

* Significantly different compared to control subjects $(p<0.05)$.
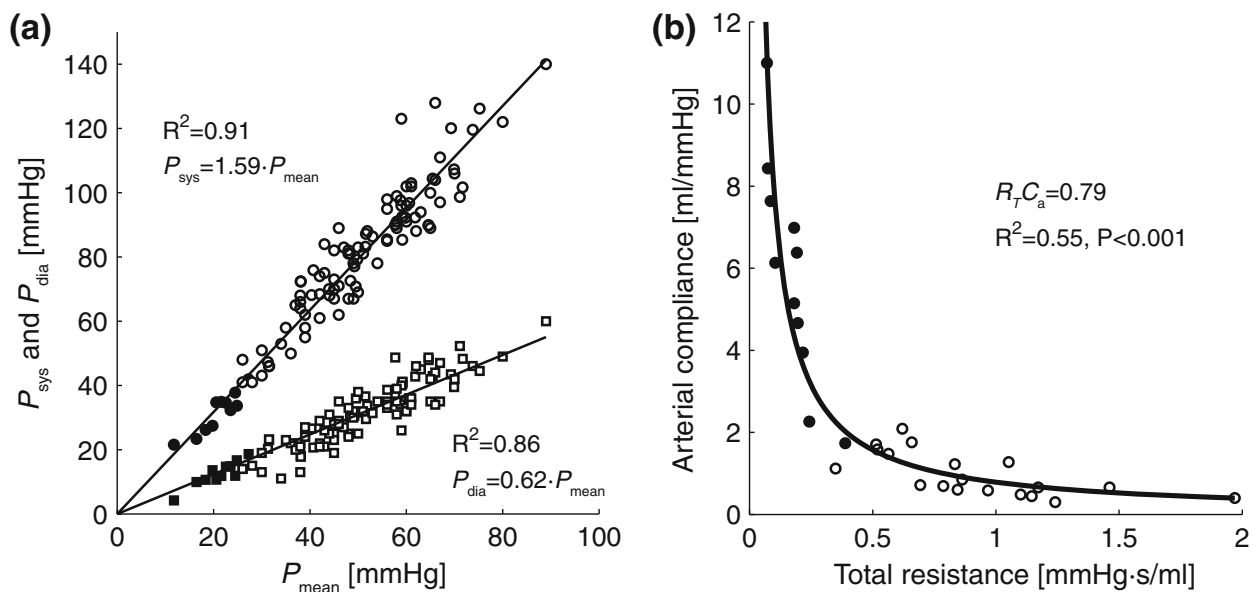

FIGURE 2. (a) Interrelationship of pulmonary artery pressures for the control subjects and PAH patients (group 1 and group $2 a+2 b$ in table 1); (b) inverse proportional relation between total vascular resistance $\left(R_{\mathrm{T}}=Z_{0}+R_{\mathrm{p}}\right)$ and arterial compliance $\left(C_{a}\right)$ for the control subjects (group 1) and subset of PAH patients (group 2a). PAH patients are indicated with open symbols, control subjects are indicated with filled symbols. The goodness-of-fit of the relationships is expressed as the coefficient of determination $\left(R^{2}\right)$.

\section{Ventricular Parameters}

Figure 4 shows the effect of isolated changes in ventricular parameters to the arterial pressures and their ratios. The figure depicts that $P_{\text {sys }}, P_{\text {dia }}, P_{\text {mean }}$ vary considerably with varying ventricular parameters, with the exception of intercept volume $V_{0}$. The effects on $K_{\text {sys }}$ and $K_{\text {dia }}$, are small except for HR that has some effect on $K_{\text {sys }}$ and $K_{\text {dia }}$. The simulations were performed assuming that left atrial pressure $\left(P_{\mathrm{la}}\right)$ is zero. However, a change of this pressure of $8 \mathrm{mmHg}$ results in linear increases of $3.5 \%$ in $K_{\text {sys }}$ and $6.2 \%$ in $K_{\text {dia }}$. 
Scenario 1: $R_{T}$ and $C_{a}$ vary independently
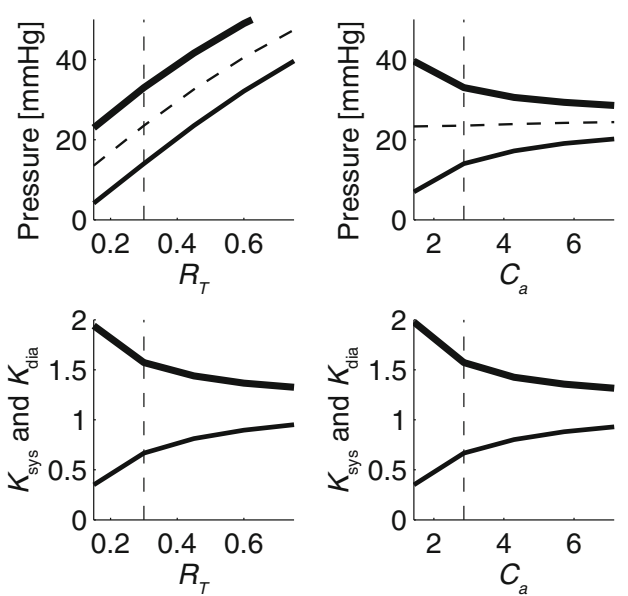

Scenario 2: $R_{T} C_{a}$-time $=$ constant
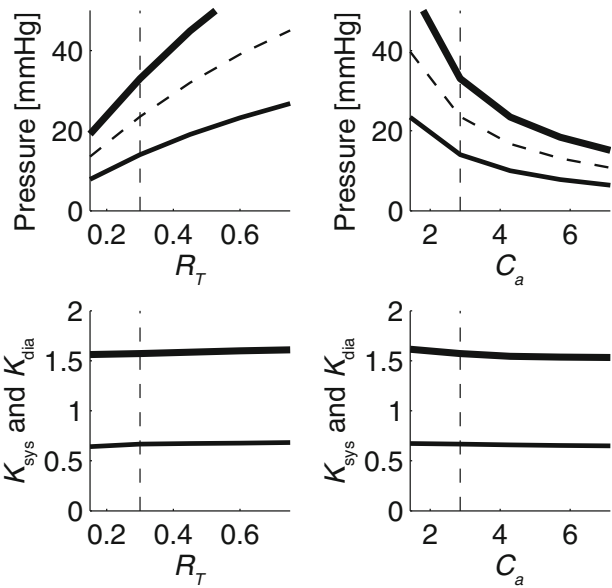

FIGURE 3. Sensitivity analysis of isolated changes of arterial parameters to $\boldsymbol{P}_{\text {sys }}$, $\boldsymbol{P}_{\text {dia }}$ and $\boldsymbol{P}_{\text {mean }}$ (upper panels), and to $K_{\text {sys }}=P_{\text {sys }} / P_{\text {mean }}$ and $K_{\text {dia }}=P_{\text {dia }} / P_{\text {mean }}$ (lower panels). The left four panels show results when total arterial resistance, $R_{\mathrm{T}}$, or arterial compliance, $C_{\mathrm{a}}$, is varied (scenario 1); the right four panels show the results when RC-time set constant in the model, i.e. an increase in $R_{\mathrm{T}}$ is accompanied by a proportional decrease in $\boldsymbol{C}_{\mathrm{a}}$ (scenario 2). For all simulations, model parameters from Group 1 in Table 1 were used, with baseline values represented by the vertical dashed lines. Systole is indicated by thick lines, diastole by thin lines, and mean pressure by dashed lines. The analysis shows that the ratios $K_{\text {sys }}$ and $K_{\text {dia }}$ are constant when RC-time is constant (bottom panels scenario 2).

\section{Contribution of Heart Rate to Pressure Proportionalities}

Sensitivity analysis indicated that changes in heart rate affected both the ratios $K_{\text {sys }}$ and $K_{\text {dia }}$, particularly at lower heart rates. This influence was further studied in experimental data of control subjects (group 1) and PAH patients (group $2 \mathrm{a}+2 \mathrm{~b}$ ). Results are shown in Fig. 5. The figure illustrates that although regression analysis did not reveal significant relations, an increase in heart rate shows a trend towards increasing $K_{\text {sys }}$ and decreasing $K_{\text {dia }}$ (thin lines). These trends seem to correspond to the trends found for simulation data (dotted lines; lines are equal to those in Fig. 4E but with a different range of heart rate). Note, however, that the simulation data is only based on baseline parameters, whereas the cardiac states in the experimental data are heterogeneous, possibly resulting in a more pronounced scatter around the trend.

\section{Simulated and Experimental Pressure Relations}

Sensitivity analysis also revealed that isolated changes of model parameters yielded either constant pressure ratios, or had effects 'mirrored about unity' on $K_{\text {sys }}$ and $K_{\text {dia }}$ (Figs. 3 and 4). This mirrored behavior implies a constant product of these ratios, which is close to unity in the simulations (e.g. for baseline values: $1.55 \cdot 0.64=0.99)$ as well in experimental data $(1.59 \cdot 0.61=0.97$; Fig. 2a). These results confirm the previous observation of Chemla et al. ${ }^{4}$ that mean pulmonary pressure is the geometric mean of systolic and diastolic pressure, since the product of $K_{\text {sys }}$ and $K_{\text {dia }}$ is close to unity so that $P_{\text {mean }}=$ $\sqrt{\left(P_{\text {sys }} \cdot P_{\text {dia }}\right)}$. This relation, however, is independent of a constant RC-time (Fig. 6).

\section{DISCUSSION}

We found that the proportional relations between systolic and diastolic pressure with mean pressure ${ }^{3,6,23}$ are based on the constant RC-time of the pulmonary arterial system, a finding previously reported by Lankhaar. ${ }^{8,9}$ In order to show this we used of an interaction model of the pulmonary circulation ${ }^{17,20}$ of which the data are presented in Figs. 3 and 4. There is negligible contribution of cardiac parameters to the proportionality of pressures is negligible, except for a small effect of heart rate (Fig. 5).

Originally, the pressure relations were reported as linear relations with a slope and intercept. Since the intercept values appeared to be small we neglected these values for simplification of the analysis. Hence, the slopes can be interpreted as the pressure ratios $K_{\text {sys }}=P_{\text {sys }} / P_{\text {mean }}$ and $K_{\text {dia }}=P_{\text {dia }} / P_{\text {mean }}$. Using our model with baseline control parameters (Group 1 in Table 1) experimentally derived proportional pressure relations could be reproduced accurately: simulated $K_{\text {sys }}$ and $K_{\text {dia }}$ were very close to our experimental ratios $(1.55$ and 0.64 vs. $1.59 \pm 0.15$ and $0.61 \pm 0.09$, Fig. 2a). The relations are also quantitatively close the relations reported by Chemla et al. ${ }^{3}$ who were the first 

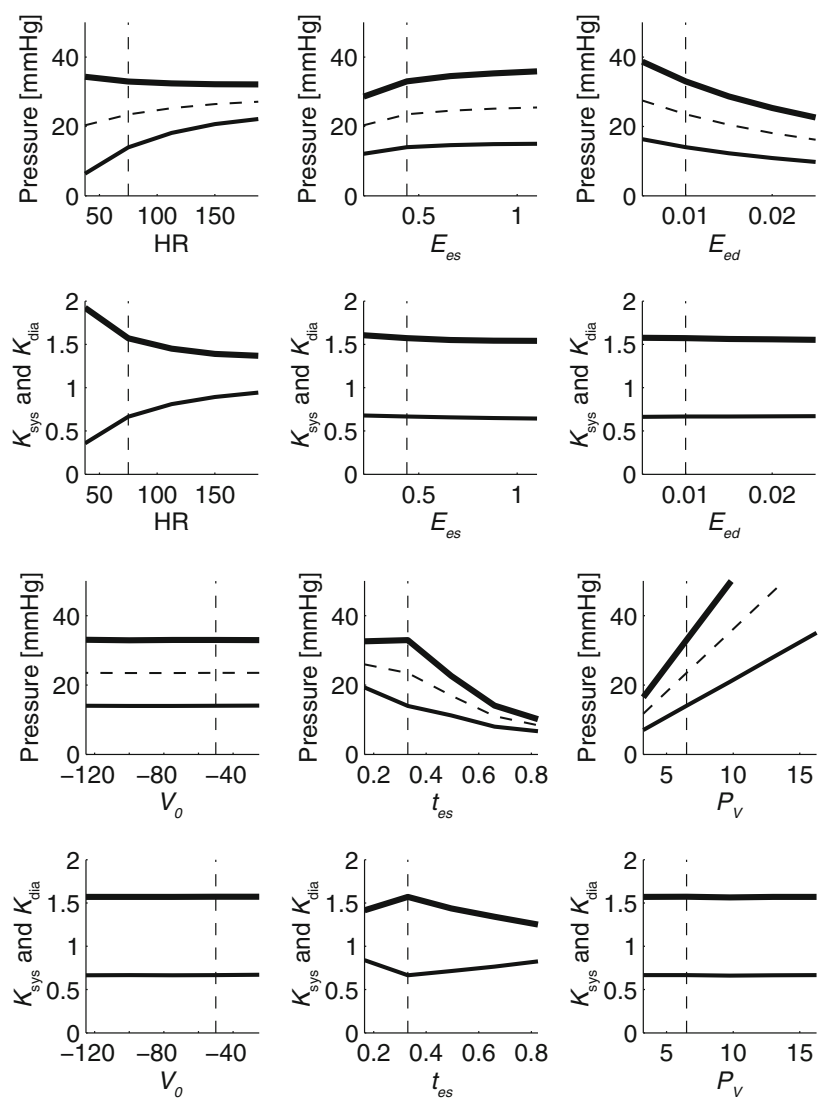

FIGURE 4. Sensitivity analysis of isolated changes of ventricular parameters on $\boldsymbol{P}_{\mathrm{sys}}, \boldsymbol{P}_{\mathrm{dia}}$ and $\boldsymbol{P}_{\text {mean }}$ (upper panels), and to $K_{\text {sys }}=P_{\text {sys }} / P_{\text {mean }}$ and $K_{\text {dia }}=P_{\text {dia }} / P_{\text {mean }}$ (lower panels). Results are shown for varying heart rate (HR), end-systolic and end-diastolic ventricular elastance $\left(E_{\mathrm{es}}\right.$ and $\left.E_{\mathrm{ed}}\right)$, intercept volume $\left(V_{0}\right)$, time to reach end-systole $\left(t_{\mathrm{es}}\right)$ and venous filling pressure $\left(\boldsymbol{P}_{\mathrm{v}}\right)$. For all simulations model parameters from group 1 in table 1 were used. Systole is indicated by thick lines, diastole by thin lines, and mean pressure by dashed lines.

to show linear pressure relations, as: $P_{\text {mean }}=0.61$. $P_{\text {sys }}+2$, which can be rewritten to $P_{\text {sys }}=1.64$. $P_{\text {mean }}-3.3$. This relation was based on high-fidelity measurements in a group of normal subjects and in PAH patients. Similar relations were derived by Friedberg et al. $^{6}$ using echocardiography $\left(P_{\text {mean }}=\right.$ $0.69 \cdot P_{\text {sys }}-0.22$ or rewritten to $P_{\text {sys }}=1.55$. $\left.P_{\text {mean }}+0.32\right)$ and by Syyed et al. $^{23}$ using high-fidelity pressure sensors $\left(P_{\text {sys }}=1.5 \cdot P_{\text {mean }}+0.45\right)$. Our results also agree with the reported relations between $P_{\text {dia }}$ and $P_{\text {mean }}$ by Syyed et al. ${ }^{23}$ who derived $P_{\text {dia }}=0.71 \cdot P_{\text {mean }}-0.66$. Therefore we can conclude that the proportionality of pressures in the pulmonary artery is a universal finding. The proportionality also implies that pulse pressure is proportional to mean pressure with a factor of about $0.99 \pm 0.23$ ( $=K_{\text {sys }}-K_{\text {dia }}$ ), i.e. pulse pressure is close to mean pressure in magnitude.

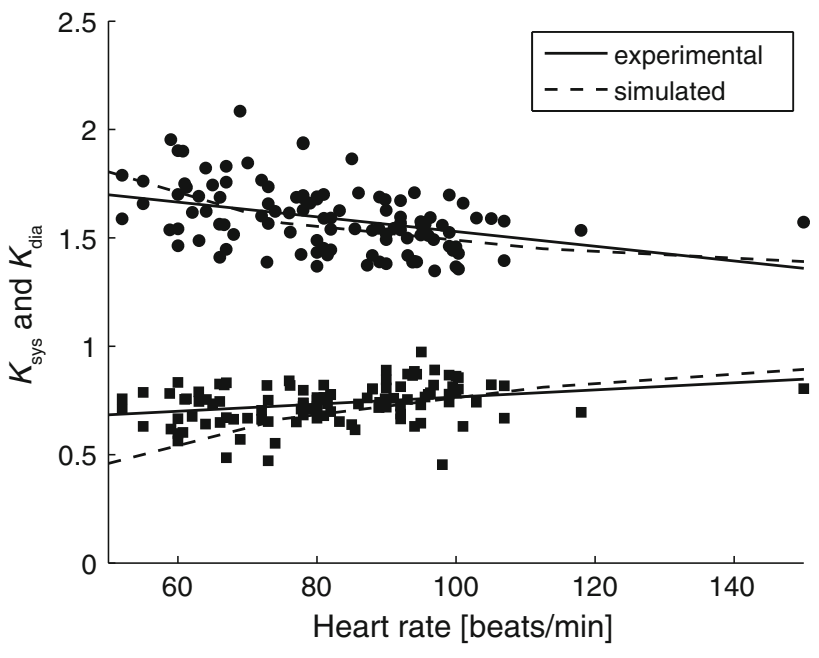

FIGURE 5. Effect of heart rate (HR) on pressure ratios $K_{\text {sys }}$ (dots) and $K_{\text {dia }}$ (squares) in the control subjects (group 1 ) and PAH patients (group $2 a+2 b$ ). Although regression analysis did not reveal significant relations, an increase in heart rate shows a trend towards increasing $K_{\text {sys }}$ and decreasing $K_{\text {dia }}$ (thin lines). The experimental data and the simulated data show similar trends (dotted lines as in Fig. 4).

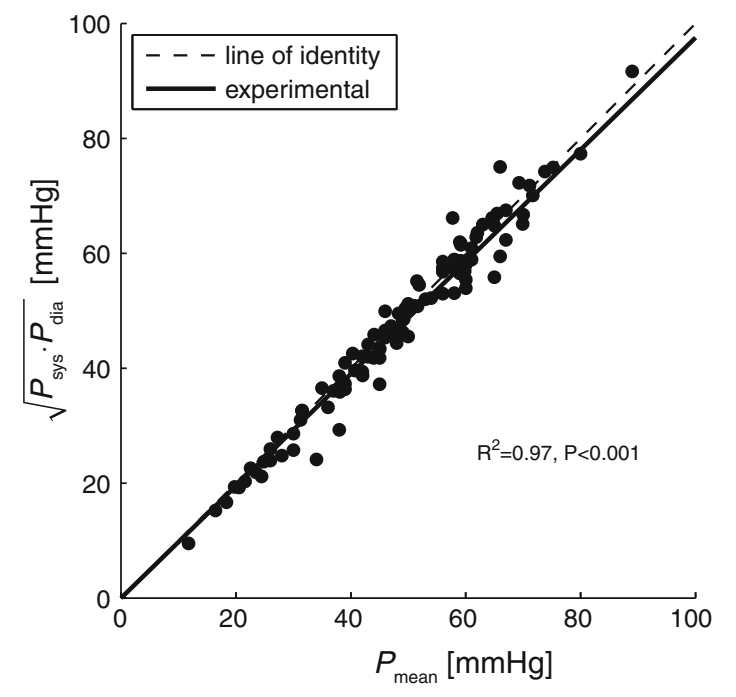

FIGURE 6. Relation between $\boldsymbol{P}_{\text {mean }}$ and $\sqrt{\boldsymbol{P}_{\text {sys }} \cdot \boldsymbol{P}_{\text {dia }}}$. Clearly, all points are close to the line of identify (gray line), implicating that $\boldsymbol{P}_{\text {mean }}$ is the geometric mean of $\boldsymbol{P}_{\text {sys }}$ and $\boldsymbol{P}_{\mathrm{dia}}{ }^{4}$

Several studies in the systemic circulation have shown that brachial and especially aortic pulse pressure are better determinants of cardiovascular events in hypertensive patients than mean pressure. ${ }^{1,5,12}$ We here show that in the pulmonary circulation, pulse pressure can be deduced from mean pulmonary pressure and is proportional to it, and, thus, does not provide more information than mean pressure.

Using our model we showed that for non-constant RC-time (scenario 1), the pressure ratios were strongly 
influenced by arterial parameters, while for constant RC-time (scenario 2) changes in arterial parameters had negligible effect on the pressure ratios. From this we conclude that the constant RC-time is a necessary condition to maintain the proportional relations reported in literature. ${ }^{3,6,23}$ The constant RC-time is also present in our experimental data (Fig. 2b) and corresponds to previous observations that $\mathrm{RC}$-time is similar in health and disease ${ }^{8}$ and also before and after treatment. $^{9}$

Sensitivity analysis indicated that ventricle parameters hardly affected pressure ratios with the exception of heart rate. However, the influence of heart rate proved to be small for the range of heart rate variation observed in supine resting conditions (Table 1 and Fig. 5). Even so, with our model the small influence could be predicted (Fig. 5), underpinning the validity of the model.

Clinical consequences of the constant RC-time are that cardiac output can be increased more when a resistance decrease is accompanied by a compliance increase (as in mild $\mathrm{PH}$ ) than when resistance alone decreases with only very small increase in compliance (as in severe PH). ${ }^{9}$ Another consequence of the constant RC-time is the recently observed proportionality in oscillatory power and total hydraulic power generated by the RV. ${ }^{16}$

In the present paper we aimed to explain the relation between the empirically derived constant RC-time and the proportionality of pulmonary artery pressures, rather than why the RC-time of the pulmonary arterial tree is constant.

We hypothesize that the constant RC-time can be explained by two mechanisms.

The first mechanism has been suggested by Sniderman et al. ${ }^{19}$ stating that an increase in resistance leads to an increase in pressure (Ohm's law), which in turn leads to a decrease in compliance as a result of the nonlinear pressure-diameter relation of pulmonary arteries. This would imply that the constant RC-time does not result from structural changes but from the elastic properties of the arteries.

The second mechanism could be based on a rather uniform distribution of resistance and compliance over the vascular bed. This is in contrast to the systemic circulation where the large arteries (in particular the aorta) are the most compliant vessels, ${ }^{7}$ but serve virtually no role in the regulation of pressure. In contrast, the small arteries and arterioles play a key role in pressure regulation and mainly determine the vascular resistance.

The hypothesis of uniform distribution of resistance and compliance in the pulmonary tree is supported by the observation that compliance of the proximal arteries is rather small compared to the total compliance of the pulmonary system. ${ }^{15}$ It has also been observed that RC-time is constant both in normal and obstructed vascular beds, implicating small variations in RC-time along artery branches.

Validation of these hypotheses requires a distributed model of the pulmonary vascular bed accounting for anatomical and mechanical vessel characteristics. Another advantage of such a model is that regional impedances and the effect of wave reflections on global RC-time can be studied.

Finally, we acknowledge some potential limitations Windkessel parameters were estimated by ignoring left atrial pressure. This results in an overestimation of peripheral resistance and thus RC-time. However, the relative variance in RC-time was comparable to Lankhaar et al., ${ }^{9}$ which may indicate only a small variation in atrial pressure and is not of relevance in the model simulations as it is just a systemic error. Another limitation regards the estimation of RV elastance parameters. These parameters could probably be estimated more accurately using multiple pressurevolume loops instead of the 'single beat method' used here, although our model reproduces the empirical pressure relations well.

\section{CONCLUSIONS}

We conclude that the constant RC-time is a necessary condition to have proportional relations between systolic and diastolic pressures with mean pulmonary artery pressure. With the constant RC-time, pressure ratios were hardly affected by variations in arterial and ventricular parameters. Although pressure ratios were sensitive to variations in heart rate, the absolute variation in heart rate was so small to have significant effects. Future studies should elucidate the physiological mechanism behind this constant value of RC-time in the pulmonary circulation.

\section{ACKNOWLEDGMENTS}

T. Kind was supported by the Netherlands Organisation for Scientific Research (NWO), Toptalent grant, project number 021.001.120. A. VonkNoordegraaf was supported by the NWO, Vidi Grant, project number 91.796.306.

\section{CONFLICTS OF INTEREST}

None. 


\section{OPEN ACCESS}

This article is distributed under the terms of the Creative Commons Attribution Noncommercial License which permits any noncommercial use, distribution, and reproduction in any medium, provided the original author(s) and source are credited.

\section{REFERENCES}

${ }^{1}$ Benetos, A., A. Rudnichi, M. Safar, and L. Guize. Pulse pressure and cardiovascular mortality in normotensive and hypertensive subjects. Hypertension 32:560-564, 1998.

${ }^{2}$ Brimioulle, S., P. Wauthy, P. Ewalenko, B. Rondelet, F. Vermeulen, F. Kerbaul, and R. Naeije. Single-beat estimation of right ventricular end-systolic pressure-volume relationship. Am. J. Physiol. Heart Circ. Physiol. 284:H1625-H1630, 2003.

${ }^{3}$ Chemla, D., V. Castelain, M. Humbert, J. L. Hebert, G. Simonneau, Y. Lecarpentier, and P. Herve. New formula for predicting mean pulmonary artery pressure using systolic pulmonary artery pressure. Chest 126:1313-1317, 2004.

${ }^{4}$ Chemla, D., V. Castelain, S. Provencher, M. Humbert, G. Simonneau, and P. Herve. Evaluation of various empirical formulas for estimating mean pulmonary artery pressure by using systolic pulmonary artery pressure in adults. Chest 135:760-768, 2009.

${ }^{5}$ Franklin, S. S., S. A. Khan, N. D. Wong, M. G. Larson, and D. Levy. Is pulse pressure useful in predicting risk for coronary heart disease? The Framingham heart study. Circulation 100:354-360, 1999.

${ }^{6}$ Friedberg, M. K., J. A. Feinstein, and D. N. Rosenthal. A novel echocardiographic Doppler method for estimation of pulmonary arterial pressures. J. Am. Soc. Echocardiogr. 19:559-562, 2006.

${ }^{7}$ Horsfield, K. Morphometry of the small pulmonary arteries in man. Circ. Res. 42:593-597, 1978.

${ }^{8}$ Lankhaar, J. W., N. Westerhof, T. J. Faes, K. M. Marques, J. T. Marcus, P. E. Postmus, and A. VonkNoordegraaf. Quantification of right ventricular afterload in patients with and without pulmonary hypertension. Am. J. Physiol. Heart Circ. Physiol. 291:H1731-H1737, 2006.

${ }^{9}$ Lankhaar, J. W., N. Westerhof, T. J. Faes, C. Tji-Joong Gan, K. M. Marques, A. Boonstra, F. G. van den Berg, P. E. Postmus, and A. Vonk-Noordegraaf. Pulmonary vascular resistance and compliance stay inversely related during treatment of pulmonary hypertension. Eur. Heart J. 29:1688-1695, 2008.

${ }^{10}$ Maughan, W. L., A. A. Shoukas, K. Sagawa, and M. L. Weisfeldt. Instantaneous pressure-volume relationship of the canine right ventricle. Circ. Res. 44:309-315, 1979.

${ }^{11}$ Milnor, W. R. Hemodynamics. Baltimore: Williams \& Wilkins, 1989.

${ }^{12}$ Mitchell, G. F., L. A. Moye, E. Braunwald, J. L. Rouleau, V. Bernstein, E. M. Geltman, G. C. Flaker, and M. A Pfeffer. Sphygmomanometrically determined pulse pressure is a powerful independent predictor of recurrent events after myocardial infarction in patients with impaired left ventricular function. SAVE investigators. Survival and ventricular enlargement. Circulation 96:4254-4260, 1997.

${ }^{13}$ Muthurangu, V., D. Atkinson, M. Sermesant, M. E. Miquel, S. Hegde, R. Johnson, R. Andriantsimiavona, A. M. Taylor, E. Baker, R. Tulloh, D. Hill, and R. S. Razavi. Measurement of total pulmonary arterial compliance using invasive pressure monitoring and MR flow quantification during MR-guided cardiac catheterization. Am. J. Physiol. Heart Circ. Physiol. 289:H1301-H1306, 2005.

${ }^{14}$ Reuben, S. R. Compliance of the human pulmonary arterial system in disease. Circ. Res. 29:40-50, 1971.

${ }^{15}$ Saouti, N., N. Westerhof, F. Helderman, J. T. Marcus, N. Stergiopulos, B. E. Westerhof, A. Boonstra, P. E. Postmus, and A. Vonk-Noordegraaf. RC-time constant of single lung equals that of both lungs together: a study in chronic thromboembolic pulmonary hypertension. Am. J. Physiol. Heart Circ. Physiol. 2009.

${ }^{16}$ Saouti, N., N. Westerhof, F. Helderman, J. T. Marcus, A. Boonstra, P. E. Postmus, and A. Vonk Noordegraaf. Right ventricular oscillatory power is a constant fraction of total power irrespective of pulmonary artery pressure. Am. J. Respir. Crit. Care Med. 2010.

${ }^{17}$ Segers, P., N. Stergiopulos, and N. Westerhof. Quantification of the contribution of cardiac and arterial remodeling to hypertension. Hypertension 36:760-765, 2000.

${ }^{18}$ Senzaki, H., C. H. Chen, and D. A. Kass. Single-beat estimation of end-systolic pressure-volume relation in humans. A new method with the potential for noninvasive application. Circulation 94:2497-2506, 1996.

${ }^{19}$ Sniderman, A. D., and D. H. Fitchett. Vasodilators and pulmonary arterial hypertension: the paradox of therapeutic success and clinical failure. Int. J. Cardiol. 20: 173-181, 1988

${ }^{20}$ Stergiopulos, N., J. J. Meister, and N. Westerhof. Determinants of stroke volume and systolic and diastolic aortic pressure. Am. J. Physiol. 270:H2050-H2059, 1996.

${ }^{21}$ Suga, H., K. Sagawa, and A. A. Shoukas. Load independence of the instantaneous pressure-volume ratio of the canine left ventricle and effects of epinephrine and heart rate on the ratio. Circ. Res. 32:314-322, 1973.

${ }^{22}$ Sunagawa, K., A. Yamada, Y. Senda, Y. Kikuchi, M. Nakamura, T. Shibahara, and Y. Nose. Estimation of the hydromotive source pressure from ejecting beats of the left ventricle. IEEE Trans. Biomed. Eng. 27:299-305, 1980.

${ }^{23}$ Syyed, R., J. T. Reeves, D. Welsh, D. Raeside, M. K. Johnson, and A. J. Peacock. The relationship between the components of pulmonary artery pressure remains constant under all conditions in both health and disease. Chest 133:633-639, 2008.

${ }^{24}$ Takeuchi, M., Y. Igarashi, S. Tomimoto, M. Odake, T. Hayashi, T. Tsukamoto, K. Hata, H. Takaoka, and H. Fukuzaki. Single-beat estimation of the slope of the end-systolic pressure-volume relation in the human left ventricle. Circulation 83:202-212, 1991.

${ }^{25}$ Westerhof, N., G. Elzinga, and P. Sipkema. An artificial arterial system for pumping hearts. J. Appl. Physiol. 31:776-781, 1971.

${ }^{26}$ Westerhof, N., J. W. Lankhaar, and B. E. Westerhof. The arterial Windkessel. Med. Biol. Eng. Comput. 47:131-141, 2008 . 